\title{
Exact Solutions of Time-Fractional KdV Equations by Using Generalized Kudryashov Method
}

\author{
Hasan Bulut, Yusuf Pandir, and Seyma Tuluce Demiray
}

\begin{abstract}
In this paper, we investigate exact solutions of time-fractional KdV equations by using generalized Kudryashov method (GKM). The time-fractional KdV equations can be reduced to nonlinear ordinary differential equation by transformation. Subsequently, GKM has been performed to obtain exact solutions of time-fractional $\mathrm{KdV}$ equations and we attain some new solutions such as soliton solutions and hyperbolic function solutions. Furthermore, we note that this method is a generalized form of classical Kudryashov Method.
\end{abstract}

Index Terms-Time-fractional $\mathrm{KdV}$ equations, generalized kudryashov method, soliton solutions, hyperbolic function solutions.

\section{INTRODUCTION}

Partial differential equations are prevalently used as models to identify numerous physical occurrences and have a very crucial role in many sciences. Korteweg-de Vries (KdV) equation, which is one type of partial differential equations, has been utilized to define a wide range of physical phenomena as a model for the evolution and interaction of nonlinear waves. It was derived as an evolution equation that conducting a one-dimensional, small amplitude, long surface gravity waves propagating in a shallow channel of water [1]. Subsequently, the KdV equation has occurred in a lot of other physical sciences such as collision-free hydromagnetic waves, stratified internal waves, ion-acoustic waves, plasma physics, lattice dynamics [2]. Some theoretical physical occurrences in the quantum mechanics domain are expressed by means of a $\mathrm{KdV}$ model. It is utilized in fluid dynamics, aerodynamics, and continuum mechanics as a model for shock wave formation, solitons, turbulence, boundary layer behaviour, and mass transport [3].

The enquiry of exact solutions to nonlinear fractional differential equations have a very crucial role in several sciences such as physics, viscoelasticity, signal processing, probability and statistics, finance, optical fibers, mechanical engineering, hydrodynamics, chemistry, solid state physics, biology, system identification, fluid mechanics, electric control theory, thermodynamics, heat transfer and fractional dynamics [4]-[6]. Exact solutions of fractional differential equations has been considered by using many methods such as the extended trial equation method [7], the modified trial

Manuscript received March 5, 2014; revised May 7, 2014.

Hasan Bulut is with Mathematics Dept., Firat University, Elazig, 23119 Turkey (e-mail: hbulut@firat.edu.tr).

Yusuf Pandir is with Mathematics Dept., Bozok University, Yozgat, 66100, Turkey (e-mail: yusufpandir@gmail.com).

Seyma Tuluce Demiray is with Mathematics Dept., Firat University, Elazig, 23119, Turkey (e-mail: seymatuluce@gmail.com). equation method [8], [9], a multiple extended trial equation method [10] and the modified kudryashov method [11], [12].

The generalized fractional $\mathrm{KdV}$ equation is given as following

$$
\frac{\partial^{\alpha} u(x, t)}{\partial t^{\alpha}}+\varepsilon[u(x, t)]^{m} \frac{\partial^{\beta} u(x, t)}{\partial x^{\beta}}+v u_{x x x}=g(x, t)
$$

For $t>0,0<\alpha, \beta \leq 1$, where $\varepsilon, v$ are constants $m=0,1,2$ and $\alpha$ and $\beta$ are parameters describing the order of the fractional time and space-derivatives, respectively. If $m=0, m=1$ and $m=2$, (1) turns into the linear fractional $\mathrm{KdV}$, nonlinear fractional $\mathrm{KdV}$ and fractional modified $\mathrm{KdV}$ $(\mathrm{mKdV})$, respectively. The function $u(x, t)$ is supposed to be a casual function of time and space, i. e. vanishing for $t<0$ and. In case of $\alpha=1$ and $\beta=1$, (1) convert into the classical mKdV equation [13].

Our purpose in this study is to present the exact solutions of time-fractional $\mathrm{KdV}$ equations [3]. In Chapter 2, we introduce the explanation of recommended method. In Chapter 3, as implementations, we find exact solutions of nonlinear time-fractional KdV equation [14]-[18]

$$
\frac{\partial^{\alpha} u(x, t)}{\partial t^{\alpha}}+6 u u_{x}+u_{x x x}=0, t>0,0<\alpha \leq 1,
$$

And time-fractional mKdV equation [13]

$$
\frac{\partial^{\alpha} u(x, t)}{\partial t^{\alpha}}+6 u^{2} u_{x}+u_{x x x}=0, t>0,0<\alpha \leq 1,
$$

where $\alpha$ is a parameter describing the order of the fractional derivative.

\section{The Generalized KudRYASHOV MethoD}

Recently, some authors have investigated Kudryashov method [19]-[21]. But, in this work, we try to constitute generalized form of Kudryashov method.

We consider the following nonlinear partial differential equation with fractional order for a function $u$ of two real variables, space $x$ and time $t$ :

$$
P\left(u, D_{t}^{\alpha} u, u_{x}, u_{x x}, u_{x x x}, \cdots\right)=0 .
$$

The basic phases of the generalized Kudryashov method is explained as follows:

Step 1. First of all, we must get the travelling wave 
solution of (1) as following form:

$$
u(x, t)=u(\eta), \eta=k x-\frac{\lambda t^{\alpha}}{\Gamma[1+\alpha]},
$$

where $k$ and $\lambda$ are arbitrary constants. Eq. (1) was converted into a nonlinear ordinary differential equation of the form:

$$
N\left(u, u^{\prime}, u^{\prime \prime}, u^{\prime \prime \prime}, \cdots\right)=0
$$

where the prime indicates differentiation with respect to $\eta$.

Step 2. Suggest that the exact solutions of (4) can be written as following form:

$$
u(\eta)=\frac{\sum_{i=0}^{N} a_{i} Q^{i}(\eta)}{\sum_{j=0}^{M} b_{j} Q^{j}(\eta)}=\frac{A[Q(\eta)]}{B[Q(\eta)]}
$$

where $Q$ is $\frac{1}{1 \pm e^{\eta}}$. We note that the function $Q$ is solution of equation given as following [19]

$$
Q_{\eta}=Q^{2}-Q
$$

Taking into consideration (5), we obtain

$$
\begin{aligned}
& u^{\prime}(\eta)=\frac{A^{\prime} \cdot Q^{\prime} \cdot B-A \cdot B^{\prime} \cdot Q^{\prime}}{B^{2}} \\
&=Q^{\prime} \cdot\left[\frac{A^{\prime} \cdot B-A \cdot B^{\prime}}{B^{2}}\right] \\
&=\left(Q^{2}-Q\right) \cdot\left[\frac{A^{\prime} \cdot B-A \cdot B^{\prime}}{B^{2}}\right], \\
& u^{\prime \prime}(\eta)=\frac{Q^{2}-Q}{B^{2}}(2 Q-1)\left(A^{\prime} \cdot B-A B^{\prime}\right) \\
&+\frac{\left(Q^{2}-Q\right)^{2}}{B^{3}}\left[B\left(A^{\prime \prime} \cdot B-A \cdot B^{\prime \prime}\right)-2 B^{\prime} \cdot A^{\prime} \cdot B+2 A\left(B^{\prime}\right)^{2}\right] .
\end{aligned}
$$

Step 3. Under the terms of proposed method, we suppose that the solution of (4) can be explained in the form of following:

$$
u(\eta)=\frac{a_{0}+a_{1} Q+a_{2} Q^{2}+\cdots+a_{N} Q^{N}+\cdots}{b_{0}+b_{1} Q+b_{2} Q^{2}+\cdots+b_{M} Q^{M}+\cdots} .
$$

To calculate the values $M$ and $N$ in (9) that is the pole order for the general solution of (4), we progress conformably as in the classical Kudryashov method on balancing the highest order nonlinear terms in (4) and we can determine a formula of $M$ and $N$. We can receive some values of $M$ and $N$.

Step 4. Replacing (5) into (4) provides a polynomial $R(\Omega)$ of $\Omega$. Establishing the coefficients of $R(\Omega)$ to zero, we acquire a system of algebraic equations. Solving this system, we can describe $\lambda$ and the variable coefficients of $a_{0}, a_{1}, a_{2}, \cdots, a_{N}, b_{0}, b_{1}, b_{2}, \cdots, b_{M}$. In this way, we attain the exact solutions to (4).

\section{Applications to the Time-Fractional KDV EQUATIONS}

In this chapter, we seek the exact solutions of nonlinear time-fractional $\mathrm{KdV}$ equation and time-fractional $\mathrm{mKdV}$ equation by using generalized Kudryashov Method.

Example 1: We handle the travelling wave solutions of (2) and we perform the transformation $u(x, t)=u(\eta)$ and $\eta=k x-\frac{\lambda t^{\alpha}}{\Gamma[1+\alpha]}$, where $k$ and $\lambda$ are constants. Then, integrating this equation with respect to $\eta$ and substituting the integration constant to zero, we reach,

$$
-\lambda u+3 k u^{2}+k^{3} u^{\prime \prime}=0 .
$$

Putting (7) and (10) into (12) and balancing the highest order nonlinear terms of $u^{\prime \prime}$ and $u^{2}$ in (12), then the following formula is procured

$$
N-M+2=2 N-2 M \Rightarrow N=M+2 .
$$

If we choose $M=1$ and $N=3$, then

$$
\begin{gathered}
u(\eta)=\frac{a_{0}+a_{1} Q+a_{2} Q^{2}+a_{3} Q^{3}}{b_{0}+b_{1} Q} \\
u^{\prime}(\eta)=\left(Q^{2}-Q\right)\left[\frac{\left.\left(a_{1}+2 a_{2} Q+3 a_{3} Q^{2}\right)\left(b_{0}+b_{1} Q\right)-b_{1}\left(a_{0}+a_{1} Q+a_{2} Q^{2}+a_{3} Q^{3}\right)\right]}{\left(b_{0}+b_{1} Q\right)^{2}}\right] \\
u^{\prime \prime}(\eta)=\frac{Q^{2}-Q}{\left(b_{0}+b_{1} Q\right)^{2}}(2 Q-1)\left[\left(a_{1}+2 a_{2} Q+3 a_{3} Q^{2}\right)\left(b_{0}+b_{1} Q\right)-b_{1}\left(a_{0}+a_{1} Q+a_{2} Q^{2}+a_{3} Q^{3}\right)\right](1 \\
+\frac{\left(Q^{2}-Q\right)^{2}}{\left(b_{0}+b_{1} Q\right)^{3}}\left[\left(b_{0}+b_{1} Q\right)^{2}\left(2 a_{2}+6 a_{3} Q\right)-2 b_{1}\left(b_{0}+b_{1} Q\right)\left(a_{1}+2 a_{2} Q+3 a_{3} Q^{2}\right)\right] \\
+\frac{\left(Q^{2}-Q\right)^{2}}{\left(b_{0}+b_{1} Q\right)^{3}}\left[2 b_{1}^{2}\left(a_{0}+a_{1} Q+a_{2} Q^{2}+a_{3} Q^{3}\right)\right]
\end{gathered}
$$

In attempt to obtain the exact solution of (2), if we take

$a_{0}=-\frac{k^{2} b_{0}}{3}, a_{1}=\frac{1}{3} k^{2}\left(6 b_{0}-b_{1}\right), a_{2}=2 k^{2}\left(b_{1}-b_{0}\right), a_{3}=-2 k^{2} b_{1}, \lambda=-k^{3}$.

and embed (17) into (14), we obtain the following solution of (2)

$$
\begin{aligned}
& -\frac{k^{2} b_{0}}{3}+\frac{1}{3} k^{2}\left(6 b_{0}-b_{1}\right)\left(\frac{1}{1 \pm e^{k+\frac{k^{2}, t^{\alpha}}{2(1+\alpha)}}}\right)+2 k^{2}\left(b_{1}-b_{0}\right)\left(\frac{1}{1 \pm e^{k+\frac{k^{3}, r^{\alpha}}{2 \Gamma(1+\alpha)}}}\right)^{2} \\
& u(x, t)=\frac{-2 k^{2} b_{1}\left(\frac{1}{1 \pm e^{k x+\frac{k^{3} \alpha}{\Gamma(1+\alpha)}}}\right)^{3}}{b_{0}+b_{1}\left(\frac{1}{1 \pm e^{k+\frac{k^{3} t^{\alpha}}{2 \Gamma(1+\alpha)}}}\right)}
\end{aligned}
$$

Using several simple transformations to this solution, we get new exact solutions to (2),

$$
u_{1}(x, t)=K\left[\left[\tanh \left(k_{1} x-\lambda_{1} t^{\alpha}\right)\right]^{2}-\frac{1}{3}\right],
$$




$$
u_{2}(x, t)=K\left[\left[\operatorname{coth}\left(k_{1} x-\lambda_{1} t^{\alpha}\right)\right]^{2}-\frac{1}{3}\right]
$$

where $K=-\frac{k^{2}}{2}, k_{1}=\frac{k}{2}$ and $\lambda_{1}=-\frac{k^{3}}{2 \Gamma(1+\alpha)}$.
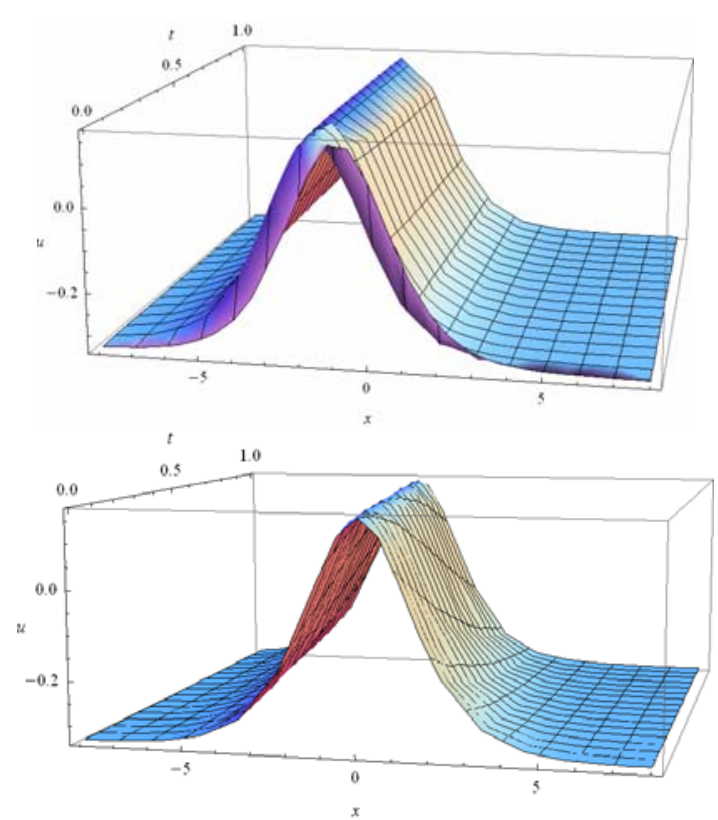

Fig. 1. Graph of the solution (19) corresponding to the values $\alpha=0.05, \alpha=0.95$ from left to right when $k=1,-8<x<8,0<t<1$.

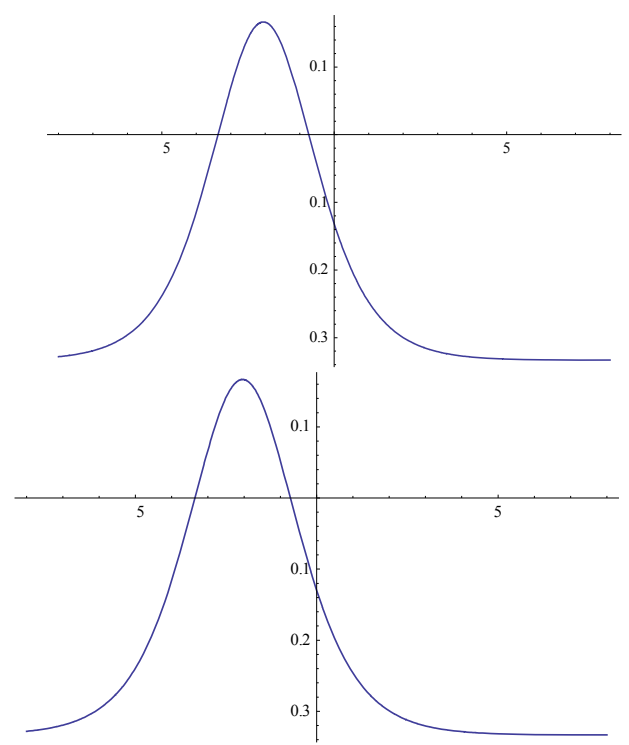

Fig. 2. Two dimensional graph of the solution (19) corresponding to the values $\alpha=0.05, \alpha=0.95$ from left to right when $k=1,-8<x<8, t=1$.

Example 2: We take the travelling wave solutions of (3) and we implement the transformation $u(x, t)=u(\eta)$ and $\eta=k x-\frac{\lambda t^{\alpha}}{\Gamma[1+\alpha]}$ where $k$ and $\lambda$ are constants.

Then, integrating this equation with respect to $\eta$ and embedding the integration constant to zero, we obtain,

$$
-\lambda u+2 k u^{3}+k^{3} u^{\prime \prime}=0 .
$$

Putting (7) and (10) into (21) and balancing the highest order nonlinear terms of $u^{\prime \prime}$ and $u^{3}$ in (21), then the following formula is procured

$$
N-M+2=3 N-3 M \Rightarrow N=M+1 .
$$

If we choose $M=1$ and $N=2$, then

$$
\begin{gathered}
u(\eta)=\frac{a_{0}+a_{1} Q+a_{2} Q^{2}}{b_{0}+b_{1} Q}, \\
u^{\prime}(\eta)=\left(Q^{2}-Q\right)\left[\frac{\left(a_{1}+2 a_{2} Q\right) \cdot\left(b_{0}+b_{1} Q\right)-b_{1}\left(a_{0}+a_{1} Q+a_{2} Q^{2}\right)}{\left(b_{0}+b_{1} Q\right)^{2}}\right], \\
u^{\prime \prime}(\eta)=\frac{Q^{2}-Q}{\left(b_{0}+b_{1} Q\right)^{2}}(2 Q-1)\left[\left(a_{1}+2 a_{2} Q\right)\left(b_{0}+b_{1} Q\right)-b_{1}\left(a_{0}+a_{1} Q+a_{2} Q^{2}+a_{3} Q^{3}\right)\right] \\
+\frac{\left(Q^{2}-Q\right)^{2}}{\left(b_{0}+b_{1} Q\right)^{3}}\left[2 a_{2}\left(b_{0}+b_{1} Q\right)^{2}-2 b_{1}\left(a_{1}+2 a_{2} Q\right)\left(b_{0}+b_{1} Q\right)+2 b_{1}^{2}\left(a_{0}+a_{1} Q+a_{2} Q^{2}\right)\right] .
\end{gathered}
$$

The exact solutions of (3) are obtained as the following; Case 1:
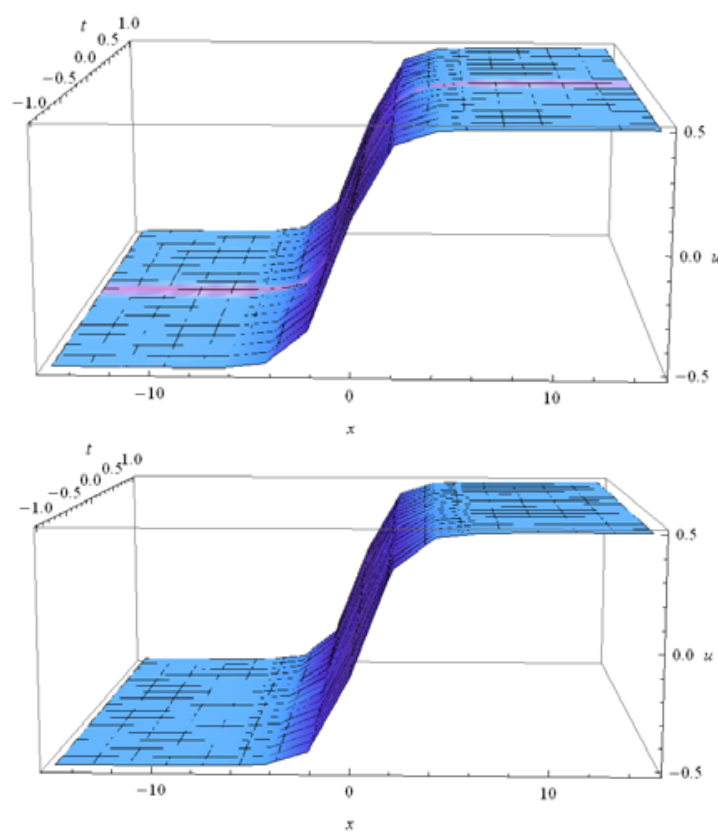

Fig. 3. Graph of the solution (28) corresponding to the values.

$$
a_{0}=\frac{1}{2} i k b_{0}, a_{1}=\frac{1}{2} i k\left(b_{1}-2 b_{0}\right), a_{2}=i k b_{1}, \lambda=-\frac{k^{3}}{2} \text {. }
$$

When we substitute (26) into (23), we get the following solution of (3)

$$
u_{1}(x, t)=\frac{\frac{1}{2} i k b_{0}+\frac{1}{2} i k\left(b_{1}-2 b_{0}\right)\left(\frac{1}{1 \pm e^{k+\frac{k_{t}^{3} t^{\alpha}}{2 \Gamma(1+\alpha)}}}\right)-i k b_{1}\left(\frac{1}{1 \pm e^{k++\frac{k_{t}^{3} t^{2}}{2 \Gamma(1+\alpha)}}}\right)^{2}}{b_{0}+b_{1}\left(\frac{1}{1 \pm e^{k x+\frac{k^{3} t^{\alpha}}{2 \Gamma(1+\alpha)}}}\right)} .
$$

Using several simple transformations to this solution, we procure new exact solution to (3),

$$
\begin{aligned}
& u_{1}(x, t)=D \tan \left[i\left(k_{1} x-\lambda_{2} t^{\alpha}\right)\right], \\
& u_{2}(x, t)=D \cot \left[i\left(k_{1} x-\lambda_{2} t^{\alpha}\right)\right],
\end{aligned}
$$


where $_{D}=\frac{k}{2}$ and $\lambda_{2}=-\frac{k^{3}}{4 \Gamma(1+\alpha)}$. $^{2}$

$\alpha=0.05, \alpha=0.85$ from left to right when $k=1,-15<x<15,-1<t<1$.

\section{Case 2:}

$$
a_{0}=\frac{1}{2} i k b_{0}, a_{1}=-\frac{1}{2} i k\left(b_{1}+2 b_{0}\right), a_{2}=0, \lambda=-\frac{k^{3}}{2}
$$

If we put (30) into (23), we obtain the following solution of (3)

$$
u_{3}(x, t)=\frac{\frac{1}{2} i k b_{0}-\frac{1}{2} i k\left(b_{1}+2 b_{0}\right)\left(\frac{1}{1 \pm e^{k x+\frac{k^{3} t^{\alpha}}{2 \Gamma(1+\alpha)}}}\right)}{b_{0}+b_{1}\left(\frac{1}{1 \pm e^{k x+\frac{k^{3} t^{\alpha}}{2 \Gamma(1+\alpha)}}}\right)} .
$$

Applying several simple transformations to this solution, we gain new exact solution to (3),

$$
\begin{aligned}
& u_{3}(x, t)=\frac{E \tanh \left(k_{1} x-\lambda_{2} t^{\alpha}\right)+F\left[\frac{1}{2}-\frac{1}{2} \tanh \left(k_{1} x-\lambda_{2} t^{\alpha}\right)\right]}{b_{0}+b_{1}\left[\frac{1}{2}-\frac{1}{2} \tanh \left(k_{1} x-\lambda_{2} t^{\alpha}\right)\right]}, \\
& u_{4}(x, t)=\frac{E \operatorname{coth}\left(k_{1} x-\lambda_{2} t^{\alpha}\right)+F\left[\frac{1}{2}-\frac{1}{2} \operatorname{coth}\left(k_{1} x-\lambda_{2} t^{\alpha}\right)\right]}{b_{0}+b_{1}\left[\frac{1}{2}-\frac{1}{2} \operatorname{coth}\left(k_{1} x-\lambda_{2} t^{\alpha}\right)\right]},
\end{aligned}
$$

where $E=\frac{1}{2} i k b_{0}$ and $F=-\frac{1}{2} i k b_{1}$.

\section{Case 3:}

$$
a_{0}= \pm \frac{1}{2} i k b_{0}, a_{1}=a_{2}=0, b_{1}=-2 b_{0}, \lambda=-\frac{k^{3}}{2} \text {. }
$$

When we replace (34) into (23), we obtain the following solution of (3)

$$
u_{5}(x, t)=\frac{ \pm \frac{1}{2} i k b_{0}}{b_{0}-2 b_{0}\left(\frac{1}{1 \pm e^{k x+\frac{k^{3} t^{\alpha}}{2 \Gamma(1+\alpha)}}}\right)} .
$$

Fulfilling several simple transformations to this solution, we get new exact solution to (3),

$$
\begin{aligned}
& u_{5}(x, t)=D \cot \left[i\left(k_{1} x-\lambda_{2} t^{\alpha}\right)\right], \\
& u_{6}(x, t)=D \tan \left[i\left(k_{1} x-\lambda_{2} t^{\alpha}\right)\right] .
\end{aligned}
$$
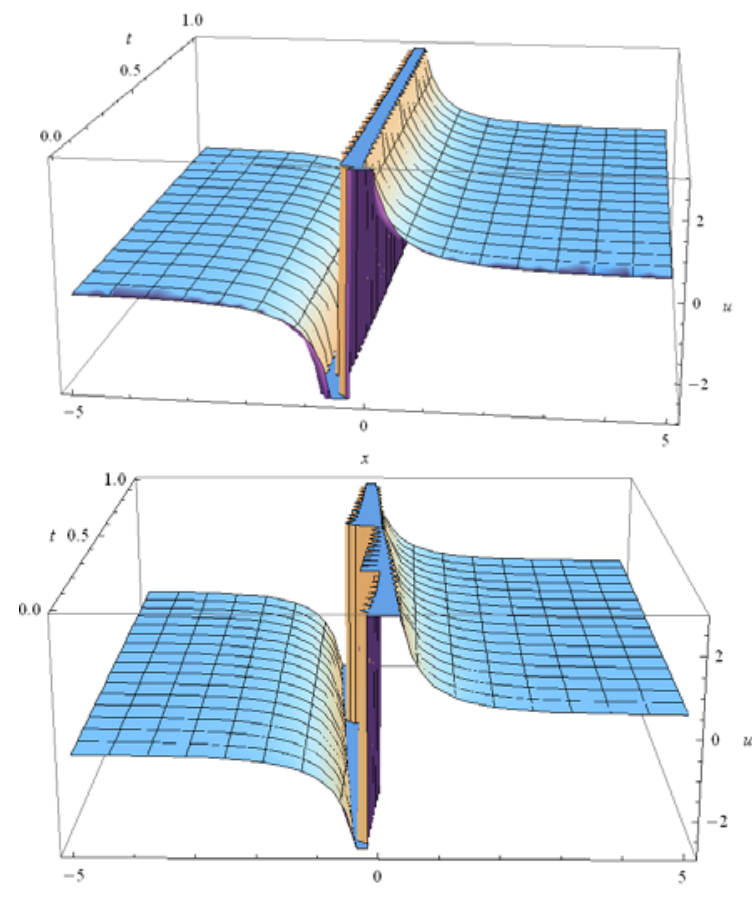

Fig. 4. Graph of the solution (36) corresponding to the values.

$\alpha=0.05, \alpha=0.85$ from left to right when $k=1,-5<x<5,0<t<1$.

\section{Case 4:}

$$
a_{0}=0, a_{1}=-2 i k b_{0}, a_{2}=2 i k b_{0}, b_{1}=-2 b_{0}, \lambda=k^{3} .
$$

If we embed (38) into (23), we find the following solution of (3)

$$
u_{7}(x, t)=\frac{-2 i k b_{0}\left(\frac{1}{1 \pm e^{k x+\frac{k^{3} t^{\alpha}}{\Gamma(1+\alpha)}}}\right)+2 i k b_{0}\left(\frac{1}{1 \pm e^{k x+\frac{k^{3} t^{\alpha}}{\Gamma(1+\alpha)}}}\right)^{2}}{b_{0}-2 b_{0}\left(\frac{1}{1 \pm e^{k x+\frac{k^{3} t^{\alpha}}{\Gamma(1+\alpha)}}}\right)}
$$

Implementing several simple transformations to this solution, we procure new exact solution to (3),

$$
\begin{aligned}
& u_{7}(x, t)=\frac{D\left[\left[\tanh \left(k_{1} x-\lambda_{3} t^{\alpha}\right)\right]^{2}-1\right]}{\tanh \left[k_{1} x-\lambda_{3} t^{\alpha}\right]}, \\
& u_{8}(x, t)=\frac{D\left[\left[\operatorname{coth}\left(k_{1} x-\lambda_{3} t^{\alpha}\right)\right]^{2}-1\right]}{\operatorname{coth}\left[k_{1} x-\lambda_{3} t^{\alpha}\right]},
\end{aligned}
$$

where $\lambda_{3}=\frac{k^{3}}{2 \Gamma(1+\alpha)}$

\section{Case 5:}

$a_{0}=i k b_{0}, a_{1}=-2 i k b_{0}, a_{2}=2 i k b_{0}, b_{1}=-2 b_{0}, \lambda=-2 k^{3}$.

If we put (42) into (23), we gain the following solution of (3) 


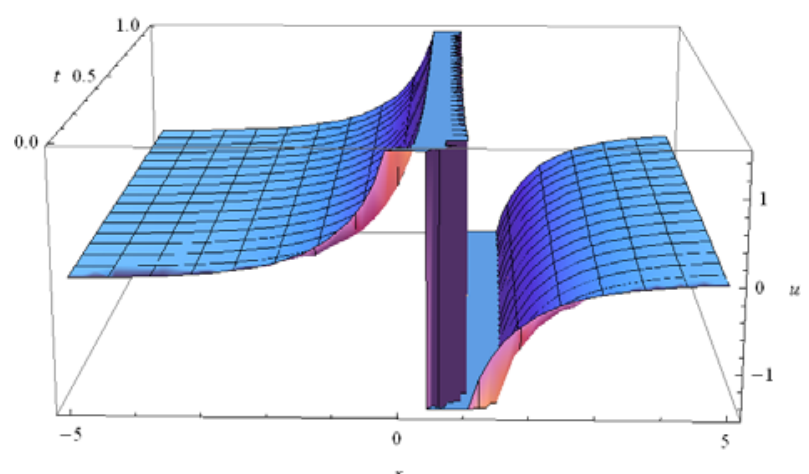

Fig. 5. Graph of the solution (40) corresponding to the values $\alpha=0.05$ and $k=1$ when $-5<x<5,0<t<1$.

$$
u_{9}(x, t)=\frac{i k b_{0}-2 i k b_{0}\left(\frac{1}{1 \pm e^{k x+\frac{k^{3} t^{\alpha}}{\Gamma(1+\alpha)}}}\right)+2 i k b_{0}\left(\frac{1}{1 \pm e^{k x+\frac{k^{3} t^{\alpha}}{\Gamma(1+\alpha)}}}\right)^{2}}{b_{0}-2 b_{0}\left(\frac{1}{1 \pm e^{k x+\frac{k^{3} t^{\alpha}}{\Gamma(1+\alpha)}}}\right)}
$$

Fulfilling several simple transformations to this solution, we attain new exact solution to (3),

$$
\begin{aligned}
& u_{9}(x, t)=\frac{D\left[1+\left[\tanh \left(k_{1} x-\lambda_{4} t^{\alpha}\right)\right]^{2}\right]}{\tanh \left[k_{1} x-\lambda_{4} t^{\alpha}\right]}, \\
& u_{10}(x, t)=\frac{D\left[1+\left[\operatorname{coth}\left(k_{1} x-\lambda_{4} t^{\alpha}\right)\right]^{2}\right]}{\operatorname{coth}\left[k_{1} x-\lambda_{4} t^{\alpha}\right]},
\end{aligned}
$$

where $\lambda_{4}=-\frac{k^{3}}{\Gamma(1+\alpha)}$.

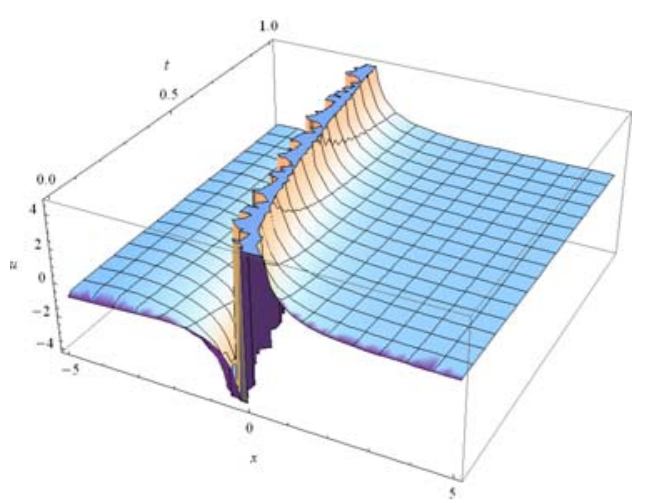

Fig. 6. Graph of the solution (44) corresponding to the values $\alpha=0.05$ and

$$
k=1 \text { when }-5<x<5,0<t<1 \text {. }
$$

We plot solution (19) of (2) in Fig. 1-2 and solutions (28), (36), (40) and (44) of (3) in Fig. 3-6, which shows the dynamics of solutions with suitable parametric choices.

Remark. The solutions of (2) and (3) were found by using generalized Kudryashov method, have been checked by means of Mathematica Release 9. To our knowledge, these solutions of (2) and (3) that we obtained in this paper, are new and are not shown in the previous literature.

\section{CONCLUSiOnS}

The Kudryashov method facilitates us in the obvious manner to produce solitary wave solutions for a broad classification of nonlinear partial differential equations. Previously, many scientists have considered Kudryashov method. But, in this paper, we try to establish generalized form of Kudryashov method. This type of method will be newly submitted to literature to construct exact solutions of nonlinear fractional differential equations.

According to these data, we can deduce that GKM plays a crucial role to reach analytical solutions of nonlinear fractional differential equations. Additionally, we note that this method is highly influential and confidential in terms of inventing new solutions such as soliton and hyperbolic function solutions. We believe that the proposed method can also be applied to other nonlinear fractional differential equations.

\section{REFERENCES}

[1] D. J. Korteweg and G. de Vries, "On the change of form of long waves advancing in a rectangular canal and on a new type of long stationary waves," Philos. Mag., vol. 39, no. 5, pp. 422-443, 1895.

[2] M. K. Fung, "KdV equation as an Euler-Poincare equation," Chin. J. Phys., vol. 35, no. 6, pp. 789-796, 1997.

[3] S. A. Elwakil, E. M. Abulwafa, M. A. Zahran, and A. A. Mahmoud, "Time-fractional $\mathrm{KdV}$ equation: formulation and solution using variational methods," Nonlinear Dyn., vol. 65, pp. 55-63, 2011.

[4] K. S. Miller and B. Ross, An Introduction to the Fractional Calculus and Fractional Differential Equations, New York, Wiley, 1993.

[5] A. A. Kilbas, H. M. Srivastava, and J. J. Trujillo, Theory and Applications of Fractional Differential Equations, San Diego, Elsevier, 2006.

[6] I. Podlubny, Fractional Differential Equations, San Diego, Academic Press, 1999.

[7] Y. Pandir, Y. Gurefe, and E. Misirli, "The extended trial equation method for some time fractional differential equations," Discrete Dynamics in Nature and Society, vol. 2013, pp. 13, 2013.

[8] H. Bulut, H. M. Baskonus, and Y. Pandir, "The extended trial equation method for some time fractional differential equations," Abstract and Applied Analysis, vol. 2013, pp. 8, 2013.

[9] Y. Pandir and Y. A. Tandogan, "Exact solutions of the time-fractional Fitzhugh-Nagumo equation," in Proc. AIP Conference, 2013, vol. 1558, pp. 1919-1922.

[10] Y. Pandir, Y. Gurefe, and E. Misirli, "A multiple extended trial equation method for the fractional Sharma-Tasso-Olver equation," in Proc. AIP Conference, 2013, vol. 1558, pp. 1927-1930.

[11] H. Bulut, Y. Pandir, and H. M. Baskonus, "Symmetrical hyperbolic Fibonacci function solutions of generalized Fisher equation with fractional order," in Proc. AIP Conference, 2013, vol. 1558, pp. 1914-1918.

[12] Y. A. Tandogan, Y. Pandir, and Y. Gurefe, "Solutions of the nonlinear differential equations by use of modified Kudryashov method," Turkish Journal of Mathematics and Computer Science, p. 7, 2013.

[13] Q. Abdulaziz, I. Hashim, and E. S. Ismail, "Approximate analytical solution to fractional modified $\mathrm{KdV}$ equations," Mathematical and Computer Modelling, vol. 49, pp. 136-145, 2009.

[14] S. Momani, "An explicit and numerical solutions of the fractional KdV equation," Mathematics and Computers in Simulation, vol. 70, pp. 110-118, 2005.

[15] Q. M. Ul-Hassan, J. Ahmad, M. Shakeel, and S. T. Mohyud-Din, "Homotopy analysis method with modified riemann-liouville derivate for solving the space- and time-fractional KDV equation," International Journal of Modern Mathematical Science, vol. 5, pp. 14-26, 2013.

[16] Z. Odibat and S. Momani, "The variational iteration method: An efficient scheme for handling fractional partial differential equations in fluid mechanics," Computers and Mathematics with Applications, vol. 58, pp. 2199-2208, 2009.

[17] L. Song and W. Wang, "Approximate rational Jacobi elliptic function solutions of the fractional differential equations via the enhanced Adomian decomposition method," Physics Letters A, vol. 374, pp. 3190-3196, 2010. 
[18] A. A. Hemeda, "Solution of fractional partial differential equations in fluid mechanics by extension of some iterative method," Abstract and Applied Analysis, vol. 2013, pp. 9, 2013.

[19] N. A. Kudryashov, "One method for finding exact solutions of nonlinear differential equations," Commun. Nonlinear Sci. Numer. Simulat., vol. 17, pp. 2248-2253, 2012.

[20] J. Lee and R. Sakthivel, "Exact travelling wave solutions for some important, nonlinear physical models," Pramana Journal of Physics, vol. 80, pp. 757-769, 2013.

[21] P. N. Ryabov, D. I. Sinelshchikov, and M. B. Kochanov, "Application of the Kudryashov method for finding exact solutions of the high order nonlinear evolution equations," Applied Mathematics and Computation, vol. 218, pp. 3965-3972, 2011.

Hasan Bulut is currently an assistant professor of Mathematics in Firat University. He has published more than 30 articles journals. His research interests include stochastic differential equations, fluid and heat mechanics, finite element method, analytical methods for nonlinear differential equations, mathematical physics, and numerical solutions of the partial differential equations, computer programming.

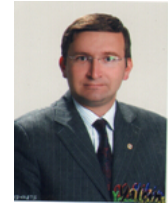

Y. Pandir is an assistant professor in Department of Mathematics at Bozok University; Yozgat (Turkey). He obtained his M.Sc. degree from Celal Bayar University and $\mathrm{Ph} . \mathrm{D}$. degree from Erciyes University, His research interests include fluid mechanics, finite element method, and analytical methods for nonlinear differential equations, mathematical physics, and numerical analysis. His research papers are published in Abstract and Applied Analysis, Applied Mathematical Sciences, International Journal of Physical Sciences, Discrete Dynamics in Nature and Society, Pramana-Journal of Physics, Physica Scripta and Journal of Mathematics.

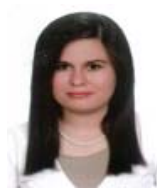

Seyma Tuluce Demiray is a research assistant (Ph.D.) in Department of Mathematics at Firat University, Elazig (Turkey). Her research interests include analytical methods for nonlinear differential equations, mathematical physics, and numerical solutions of the partial differential equations, computer programming. 